\title{
Modeling Saturated Diagnosis and Vaccination in Reducing HIV/AIDS Infection
}

\author{
Can Chen and Yanni Xiao \\ Department of Applied Mathematics, Xian Jiaotong University, Xian 710049, China \\ Correspondence should be addressed to Yanni Xiao; yxiao@mail.xjtu.edu.cn
}

Received 18 January 2014; Accepted 26 February 2014; Published 30 March 2014

Academic Editor: Kaifa Wang

Copyright ( 2014 C. Chen and Y. Xiao. This is an open access article distributed under the Creative Commons Attribution License, which permits unrestricted use, distribution, and reproduction in any medium, provided the original work is properly cited.

\begin{abstract}
A mathematical model is proposed to consider the effects of saturated diagnosis and vaccination on HIV/AIDS infection. By employing center manifold theory, we prove that there exists a backward bifurcation which suggests that the disease cannot be eradicated even if the basic reproduction number is less than unity. Global stability of the disease-free equilibrium is investigated for appropriate conditions. When the basic reproduction number is greater than unity, the system is uniformly persistent. The proposed model is applied to describe HIV infection among injecting drug users (IDUs) in Yunnan province, China. Numerical studies indicate that new cases and prevalence are sensitive to transmission rate, vaccination rate, and vaccine efficacy. The findings suggest that increasing vaccination rate and vaccine efficacy and enhancing interventions like reducing share injectors can greatly reduce the transmission of HIV among IDUs in Yunnan province, China.
\end{abstract}

\section{Introduction}

Acquired immunodeficiency syndrome (AIDS) is spreading rapidly in the world ever since it was firstly detected in 1981 and continues to threaten the health of human seriously, especially among sex workers and injecting drug users. Furthermore, AIDS also influences the economy of many countries which has attracted great attention of governments. For such a severe scenario, the governments have taken intervention measures to reduce HIV transmission.

Mathematical models play a vital role in gaining a quantitative insight into HIV transmission dynamics and suggesting the effective control strategies. In order to study the effect of various intervention strategies on HIV transmission, extensive mathematical models have been formulated. Traditionally, models of HIV/AIDS dynamics often incorporate staged progression (see, e.g., [1-3]), but these did not include any control measures. Hyman et al. [4] extended these models to consider screening and contact tracing and discussed which strategy would slow infectiousness. Compartmental models with staged progression that incorporate the imperfect vaccine were constructed in [5] to predict HIV epidemic, but they did not consider diagnosis. Elbasha and Gumel [6] considered that a proportion of new recruits are vaccinated and upon becoming infected with HIV, susceptible and vaccinated individuals enter the classes of infected and vaccine infected people, separately. They showed the existence of backward bifurcation via numerical simulations. Sharomi et al. [7] explored the role of the choice of incidence function in HIV models formulated in [6] and obtained that the phenomenon of backward bifurcation can be removed by substituting the standard incidence function with a mass action incidence. In South Africa, testing and screening campaign was launched for HIV; Nyabadza and Mukandavire [8] analyzed their effects by developing HIV models. More recently, a model of HIV/AIDS with diagnosis was presented in [9]. The authors estimated parameter values and predicted its transmission in China in the next few years.

The majority of mathematical models consider only one control strategy, vaccination or diagnosis, for instance [5, 9]; however, curbing HIV/AIDS infection needs comprehensive strategies, since, under the serious threat of HIV, it may be more rational to adopt various measures for different high risk groups. These motivate us to consider two combined intervention measures, vaccination and diagnosis. Furthermore, due to the limited resources, we then choose a nonlinear function which can be used to describe saturation effect. We use a parameter $h$, representing the half saturation 
constant, in the diagnosis function to measure the effect of HIV individuals being late for diagnosis [10]. When the number of infected individuals $I$ is low, the number of actual per capita diagnosed individuals is proportional to $I$, whereas when the number of infected individuals $I$ is sufficiently large, there is a saturation effect which makes the number of diagnosed individuals approach to be constant due to the limitation of human and economic power. The number of new diagnosed cases per unit time is saturated with the total infected population.

The paper is organized as follows. The model is formulated in Section 2. The existence of backward bifurcation and the stability of the disease-free equilibrium are discussed in Section 3. In Section 4, uniform persistence of the model is investigated. Numerical simulation results are concluded in Section 5. In Section 6, we give a brief summary.

\section{The Model}

The model describes the spread of HIV/AIDS in a high risk population. The total high risk population size at time $t$, denoted by $(N(t))$, is subdivided into susceptible individuals $(S(t))$, vaccinated susceptible individuals $(V(t))$, HIV infected but not yet diagnosed individuals $(I(t))$, diagnosed HIVpositive individuals $(D(t))$, and those who have developed AIDS $(A(t))$, so that $N(t)=S(t)+V(t)+I(\mathrm{t})+D(t)+A(t)$.

The equations of the model are

$$
\begin{aligned}
S^{\prime} & =\Pi+\omega V-\lambda(t) S-\xi S-\mu S, \\
V^{\prime} & =\xi S-(1-\epsilon) \lambda(t) V-\omega V-\mu V, \\
I^{\prime} & =\lambda(t)(S+(1-\epsilon) V)-\sigma_{1} I-\frac{q I}{1+h I}-\mu I, \\
D^{\prime} & =\frac{q I}{1+h I}-\sigma_{2} D-\mu D, \\
A^{\prime} & =\sigma_{1} I+\sigma_{2} D-(\mu+\psi) A,
\end{aligned}
$$

where the incidence rate $\lambda(t)=\beta\left(\left(I(t)+\eta_{1} D(t)+\right.\right.$ $\left.\left.\eta_{2} A(t)\right) / N(t)\right), \beta$ denotes the transmission rate, and $\eta_{1}$ and $\eta_{2}$ illustrate the modification factors in the transmission coefficient of diagnosed HIV-positive individuals and AIDS patients, respectively. People enter into the susceptible class at a rate $\Pi$, become infected at a rate $\lambda(t) S$, and become vaccinated at a rate $\xi$. Also $\mu$ is the natural death rate; $\omega$ denotes the waning rate of vaccine; $\epsilon$ represents the vaccine efficacy; $q$ is the diagnosis rate; $\sigma_{1}$ and $\sigma_{2}$ are the progression rate to diagnose HIV-positive individuals and AIDS patients, respectively; $\psi$ is the disease-induced death rate.

Since the model monitors change in the human population, the variables and parameters are assumed to be nonnegative for all $t \geq 0$. The system will be analyzed in a suitable feasible region $\Omega \subseteq R_{+}^{5}$, where $\Omega=\{(S, V, I, D, A) \in$ $\left.R_{+}^{5} \mid S+V+I+D+A \leq \Pi / \mu\right\}$. We can easily prove that the solutions of system (1) with nonnegative initial conditions remain nonnegative, and the feasible region $\Omega$ is positively invariant and attracting with respect to system (1) for all $t>0$.

\section{Model Analysis}

3.1. Disease-Free Equilibrium and the Basic Reproduction Number. Model (1) has a disease-free equilibrium (DFE), obtained by setting the right-hand sides of system (1) to zero, represented as

$$
\begin{aligned}
E_{0}:(\bar{S}, \bar{V}, \bar{I}, \bar{D}, \bar{A}) & =\left(\frac{\Pi(\mu+\omega)}{\mu(\mu+\xi+\omega)}, \frac{\Pi \xi}{\mu(\mu+\xi+\omega)}, 0,0,0\right), \\
\bar{N} & =\bar{S}+\bar{V}+\bar{I}+\bar{D}+\bar{A}=\frac{\Pi}{\mu} .
\end{aligned}
$$

Following [11], the reproduction number can be established by using the next generation operator approach. The matrices for new infection and transition terms, respectively, given by $F$ and $V$, are

$$
\begin{aligned}
& F=\left(\begin{array}{ccc}
\beta m & \eta_{1} \beta m & \eta_{2} \beta m \\
0 & 0 & 0 \\
0 & 0 & 0
\end{array}\right), \\
& V=\left(\begin{array}{ccc}
\sigma_{1}+q+\mu & 0 & 0 \\
-q & \sigma_{2}+\mu & 0 \\
-\sigma_{1} & -\sigma_{2} & \mu+\psi
\end{array}\right),
\end{aligned}
$$

where $m=(\bar{S}+(1-\epsilon) \bar{V}) / \bar{N}=(\mu+\omega+(1-\epsilon) \xi) /(\mu+\omega+\xi)$.

Denote by $R_{0}$ the basic reproduction number as

$$
\begin{aligned}
R_{0} & =\rho\left(F V^{-1}\right) \\
& =\frac{\beta m}{\sigma_{1}+q+\mu}\left(1+\frac{\eta_{1} q}{\sigma_{2}+\mu}+\frac{\eta_{2}}{\mu+\psi}\left(\frac{\sigma_{2} q}{\sigma_{2}+\mu}+\sigma_{1}\right)\right),
\end{aligned}
$$

that is, the spectral radius of the next generation matrix $F V^{-1}$. Biologically speaking, $R_{0}$ is the average number of new secondary infections generated by a single HIV infected individual, introduced into a susceptible population in which some individuals have been vaccinated.

3.2. Existence of Backward Bifurcation. Employing the center manifold theory as described in [12], we investigate the existence of backward bifurcation. In order to apply the center manifold theory, we make the following changes of variables:

$$
S=x_{1}, \quad V=x_{2}, \quad I=x_{3}, \quad D=x_{4}, \quad A=x_{5},
$$

so that $N=\sum_{n=1}^{5} x_{n}$. We now use the vector notation $X=$ $\left(x_{1}, x_{2}, x_{3}, x_{4}, x_{5}\right)^{T}$, where $(\cdot)^{T}$ denotes a matrix transpose. System (1) can then be written as $\dot{X}=F=\left(f_{1}, f_{2}, f_{3}, f_{4}, f_{5}\right)^{T}$, so that

$$
\begin{aligned}
& \dot{x}_{1}(t)=f_{1}=\Pi+\omega x_{2}-\lambda x_{1}-\xi x_{1}-\mu x_{1}, \\
& \dot{x}_{2}(t)=f_{2}=\xi x_{1}-(1-\epsilon) \lambda x_{2}-\omega x_{2}-\mu x_{2},
\end{aligned}
$$




$$
\begin{aligned}
& \dot{x}_{3}(t)=f_{3}=\lambda\left(x_{1}+(1-\epsilon) x_{2}\right)-\left(\sigma_{1}+\frac{q}{1+h x_{3}}+\mu\right) x_{3}, \\
& \dot{x}_{4}(t)=f_{4}=\frac{q x_{3}}{1+h x_{3}}-\left(\sigma_{2}+\mu\right) x_{4}, \\
& \dot{x}_{5}(t)=f_{5}=\sigma_{1} x_{3}+\sigma_{2} x_{4}-(\psi+\mu) x_{5},
\end{aligned}
$$

where $\lambda=\beta\left(\left(x_{3}+\eta_{1} x_{4}+\eta_{2} x_{5}\right) / N\right)$.
If $\beta$ is taken as the bifurcation parameter and we consider the case $R_{0}=1$, solving for $\beta$ gives $\beta=\beta^{*}$; that is,

$$
\beta^{*} m\left(1+\frac{\eta_{1} q}{\sigma_{2}+\mu}+\frac{\eta_{2}}{\mu+\psi}\left(\frac{\sigma_{2} q}{\sigma_{2}+\mu}+\sigma_{1}\right)\right)=\sigma_{1}+q+\mu
$$

First of all, observe that the eigenvalues of the Jacobian matrix $J\left(E_{0}\right)$ at $\beta=\beta^{*}[13]$, that is, $\left.J\left(E_{0}\right)\right|_{\beta=\beta^{*}}$,

$$
\left.J\left(E_{0}\right)\right|_{\beta=\beta^{*}}=\left(\begin{array}{ccccc}
-\mu-\xi & \omega & -\beta^{*} \frac{\bar{S}}{\bar{N}} & -\eta_{1} \beta^{*} \frac{\bar{S}}{\bar{N}} & -\eta_{2} \beta^{*} \frac{\bar{S}}{\bar{N}} \\
\xi & -\mu-\omega & -\beta^{*}(1-\epsilon) \overline{\bar{N}} & -\eta_{1} \beta^{*}(1-\epsilon) \frac{\bar{V}}{\bar{N}} & -\eta_{2} \beta^{*}(1-\epsilon) \frac{\bar{V}}{\bar{N}} \\
0 & 0 & \beta^{*} m-\sigma_{1}-q-\mu & \eta_{1} \beta^{*} m & \eta_{2} \beta^{*} m \\
0 & 0 & q & -\sigma_{2}-\mu & 0 \\
0 & 0 & \sigma_{1} & \sigma_{2} & -\mu-\psi
\end{array}\right),
$$

are given by

$$
\lambda_{1}=-\mu, \quad \lambda_{2}=-(\mu+\omega+\xi), \quad \lambda_{3}=0 .
$$

The other two eigenvalues satisfy the following equation:

$$
\lambda^{2}+b \lambda+c=0
$$

where

$$
\begin{aligned}
b= & \left(\sigma_{2}+\mu\right)+(\mu+\psi)+\left(\sigma_{1}+q+\mu\right)-\beta^{*} m, \\
c= & \left(\sigma_{2}+\mu\right)(\mu+\psi)+\left(\sigma_{2}+\mu\right)\left(\sigma_{1}+q+\mu-\beta^{*} m\right) \\
& +(\mu+\psi)\left(\sigma_{1}+q+\mu-\beta^{*} m\right)-\eta_{1} q \beta^{*} m-\sigma_{1} \eta_{2} \beta^{*} m .
\end{aligned}
$$

$$
\begin{aligned}
= & \left(\sigma_{2}+\mu\right)(\mu+\psi) \\
& \times\left\{1+\beta^{*} m\left[\frac{\eta_{2}}{(\mu+\psi)^{2}}\left(\frac{\sigma_{2} q}{\sigma_{2}+\mu}+\sigma_{1}\right)\right.\right.
\end{aligned}
$$

$$
\left.\left.+\frac{\eta_{1} q}{\left(\sigma_{2}+\mu\right)^{2}}+\frac{\eta_{2} \sigma_{2} q}{(\mu+\psi)\left(\sigma_{2}+\mu\right)^{2}}\right]\right\} \text {. }
$$

Clearly, $b$ and $c$ are positive. Equation (10) has two roots with negative real parts. Hence, $\lambda_{3}=0$ is a simple zero eigenvalue and all other eigenvalues have negative real parts. The assumptions in [12] are satisfied. Therefore, the center manifold theory can be used to analyze the dynamics of system (1) near $\beta=\beta^{*}$ (or, equivalently, $R_{0}=1$ ). The Jacobian matrix of system (1) at $\beta=\beta^{*}$ has a right eigenvector $w$, given by $w=\left(w_{1}, w_{2}, w_{3}, w_{4}, w_{5}\right)^{T}$. And it can be computed from the system $\left(\left.J\left(E_{0}\right)\right|_{\beta=\beta^{*}}\right) \cdot w=0$; that is,

$$
\begin{gathered}
0=-(\mu+\xi) w_{1}+\omega w_{2}-\beta^{*} \frac{\overline{x_{1}}}{\bar{N}} w_{3}-\eta_{1} \beta^{*} \frac{\overline{x_{1}}}{\bar{N}} w_{4} \\
-\eta_{2} \beta^{*} \frac{\overline{x_{1}}}{\bar{N}} w_{5}, \\
0=\xi w_{1}-(\mu+\omega) w_{2}-(1-\epsilon) \beta^{*} \frac{\overline{x_{2}}}{\bar{N}} w_{3} \\
-(1-\epsilon) \eta_{1} \beta^{*} \frac{\overline{x_{2}}}{\bar{N}} w_{4}-(1-\epsilon) \eta_{2} \beta^{*} \frac{\overline{x_{2}}}{\bar{N}} w_{5}, \\
0\left(\beta^{*} m-\sigma_{1}-q-\mu\right) w_{3}+\eta_{1} \beta^{*} m w_{4}+\eta_{2} \beta^{*} m w_{5}, \\
0=q w_{3}-\left(\sigma_{2}+\mu\right) w_{4}, \\
0=\sigma_{1} w_{3}+\sigma_{2} w_{4}-(\mu+\psi) w_{5} ;
\end{gathered}
$$


from (13), we derive the following solutions:

$$
\begin{aligned}
& w_{1}=-\frac{(\mu+w)^{2}+(1-\epsilon) w \xi}{\mu(\mu+w+\xi)^{2}} \beta^{*}\left(w_{3}+\eta_{1} w_{4}+\eta_{2} w_{5}\right)<0, \\
& w_{2}=-\frac{\xi(\mu+w+(1-\epsilon)(\mu+\xi))}{\mu(\mu+w+\xi)^{2}} \beta^{*}\left(w_{3}+\eta_{1} w_{4}+\eta_{2} w_{5}\right)
\end{aligned}
$$$$
<0 \text {, }
$$

$$
\begin{gathered}
w_{3}=w_{3}>0, \quad w_{4}=\frac{q}{\sigma_{2}+\mu} w_{3}>0, \\
w_{5}=\frac{\sigma_{1} w_{3}+\sigma_{2} w_{4}}{\mu+\psi}>0 .
\end{gathered}
$$

The left eigenvector of $\left.J\left(E_{0}\right)\right|_{\beta=\beta^{*}}$ is $v$, denoted by $v=$ $\left(v_{1}, v_{2}, v_{3}, v_{4}, v_{5}\right)$. And it can be computed from the system $\left(\left.J\left(E_{0}\right)\right|_{\beta=\beta^{*}}\right)^{T} \cdot v=0$; that is,

$$
\begin{gathered}
0=-(\mu+\xi) v_{1}+\xi v_{2}, \\
0=\omega v_{1}-(\mu+\omega) v_{2}, \\
0=-\beta^{*} \frac{\overline{x_{1}}}{\bar{N}} v_{1}-(1-\epsilon) \beta^{*} \frac{\overline{x_{2}}}{\bar{N}} v_{2}+\left(\beta^{*} m-\sigma_{1}-q-\mu\right) v_{3} \\
+q v_{4}+\sigma_{1} v_{5}, \\
0=-\eta_{1} \beta^{*} \frac{\overline{x_{1}}}{\bar{N}} v_{1}-(1-\epsilon) \eta_{1} \beta^{*} \frac{\overline{x_{2}}}{\bar{N}} v_{2}+\eta_{1} \beta^{*} m v_{3} \\
\quad-\left(\sigma_{2}+\mu\right) v_{4}+\sigma_{2} v_{5}, \\
0=-\eta_{2} \beta^{*} \frac{\overline{x_{1}}}{\bar{N}} v_{1}-(1-\epsilon) \eta_{2} \beta^{*} \frac{\overline{x_{2}}}{\bar{N}} v_{2}+\eta_{2} \beta^{*} m v_{3} \\
\quad-(\mu+\psi) v_{5},
\end{gathered}
$$

with the following solutions:

$$
\begin{gathered}
v_{1}=0, \quad v_{2}=0, \quad v_{3}=v_{3}>0, \\
v_{4}=\frac{\beta^{*} m}{\sigma_{2}+\mu}\left(\eta_{1}+\frac{\sigma_{2} \eta_{2}}{\mu+\psi}\right) v_{3}>0, \quad v_{5}=\frac{\eta_{2} \beta^{*} m}{\mu+\psi} v_{3}>0 .
\end{gathered}
$$

The local bifurcation analysis near $\beta=\beta^{*}\left(R_{0}=1\right)$ is then determined by the signs of two associated constants, denoted by $a$ and $b$, respectively, as

$$
\begin{gathered}
a=\sum_{k, i, j=1}^{5} v_{k} w_{i} w_{j} \frac{\partial^{2} f_{k}}{\partial x_{i} \partial x_{j}}(0,0), \\
b=\sum_{k, i=1}^{5} v_{k} w_{i} \frac{\partial^{2} f_{k}}{\partial x_{i} \partial \beta^{*}}(0,0) .
\end{gathered}
$$

The computations of $a$ and $b$ are done as follows: for system (6) the associated nonzero partial derivatives of $F$ at the disease-free equilibrium are

$$
\begin{gathered}
\frac{\partial^{2} f_{3}}{\partial x_{1} \partial x_{3}}=\frac{\beta^{*}}{\bar{N}}(1-m), \quad \frac{\partial^{2} f_{3}}{\partial x_{1} \partial x_{4}}=\frac{\eta_{1} \beta^{*}}{\bar{N}}(1-m), \\
\frac{\partial^{2} f_{3}}{\partial x_{1} \partial x_{5}}=\frac{\eta_{2} \beta^{*}}{\bar{N}}(1-m), \quad \frac{\partial^{2} f_{3}}{\partial x_{2} \partial x_{3}}=\frac{\beta^{*}}{\bar{N}}(1-\epsilon-m), \\
\frac{\partial^{2} f_{3}}{\partial x_{2} \partial x_{4}}=\frac{\eta_{1} \beta^{*}}{\bar{N}}(1-\epsilon-m), \\
\frac{\partial^{2} f_{3}}{\partial x_{3} \partial x_{3}}=-\frac{\eta_{2} \beta^{*}}{\bar{N}}(1-\epsilon-m), \\
\frac{\partial^{2} f_{3}}{\partial x_{3} \partial x_{5}}=-\frac{\beta^{2} f_{3}}{\partial x_{3} \partial x_{4}}=-\frac{\beta^{*}\left(1+\eta_{1}\right)}{\bar{N}} m, \\
\frac{\partial^{2} f_{3}}{\partial x_{4} \partial x_{5}}=-\frac{\partial^{2} f_{3}}{\partial x_{4} \partial x_{4}}=-\frac{2 \eta_{1} \beta^{*}}{\bar{N}} m, \\
\frac{\partial^{2} f_{3}}{\partial x_{3} \partial \beta^{*}}=m, \quad \frac{\left.\eta_{2}\right)}{\partial x_{4} \partial \beta^{*}}=\eta_{1} m, \quad \frac{\partial^{2} f_{3}}{\partial x_{5} \partial x_{5}}=-\frac{2 \eta_{2} \beta^{*}}{\bar{N}} m, \\
\frac{\partial^{2} f_{4}}{\partial x_{3} \partial x_{3}}=-2 q h, \\
\frac{\eta_{3}}{\partial x_{5} \partial \beta^{*}}=\eta_{2} m .
\end{gathered}
$$

Substituting (18) into (17), we get

$$
\begin{aligned}
& a=\frac{2 \beta^{*}}{\bar{N}} v_{3}\{\left(w_{3}+\eta_{1} w_{4}+\eta_{2} w_{5}\right) \\
& \times\left(\beta^{*}\left(w_{3}+\eta_{1} w_{4}+\eta_{2} w_{5}\right) \frac{(1-\epsilon) \epsilon \xi}{(\mu+w+\xi)^{2}}\right. \\
&\left.\left.-m\left(w_{3}+w_{4}+w_{5}\right)\right)\right\} \\
&+2 q h w_{3}^{2} v_{3}\left(1-\frac{\beta^{*} m}{\sigma_{2}+\mu}\left(\eta_{1}+\frac{\sigma_{2} \eta_{2}}{\mu+\psi}\right)\right) \\
& b=v_{3}\left(w_{3}+\eta_{1} w_{4}+\eta_{2} w_{5}\right) m .
\end{aligned}
$$

From $[14,15]$, we know that if $a>0, b>0$, there exists a backward bifurcation. Since the bifurcation coefficient, $b$, is always positive, then we establish the following result.

Theorem 1. If $a>0$, system (1) exhibits a backward bifurcation when $R_{0}=1$.

Due to existence of backward bifurcation we know that, for positive $a$, there exists another critical value $R_{c}$, which is less than unity, for model (1). Moreover, there is no endemic equilibrium for $R_{0}<R_{c}$; there are two distinct endemic 


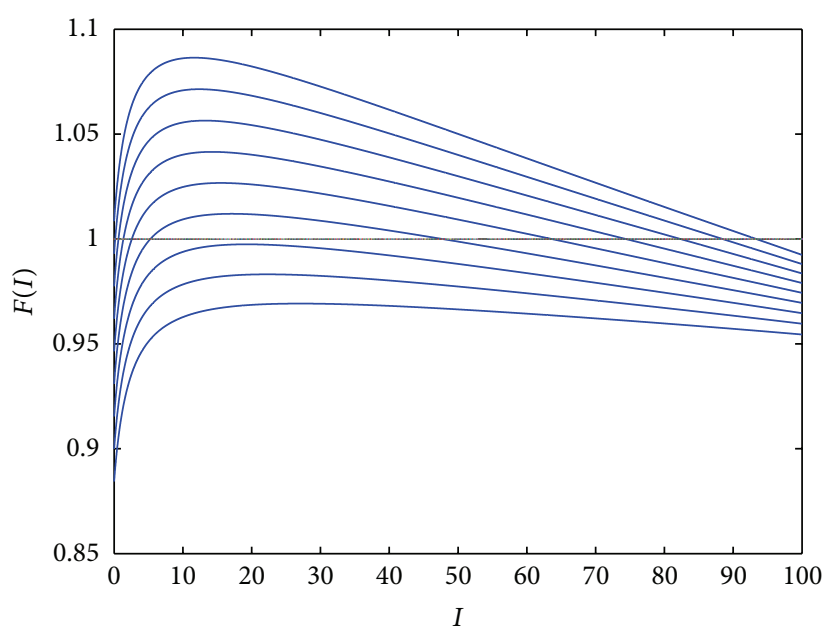

(a)

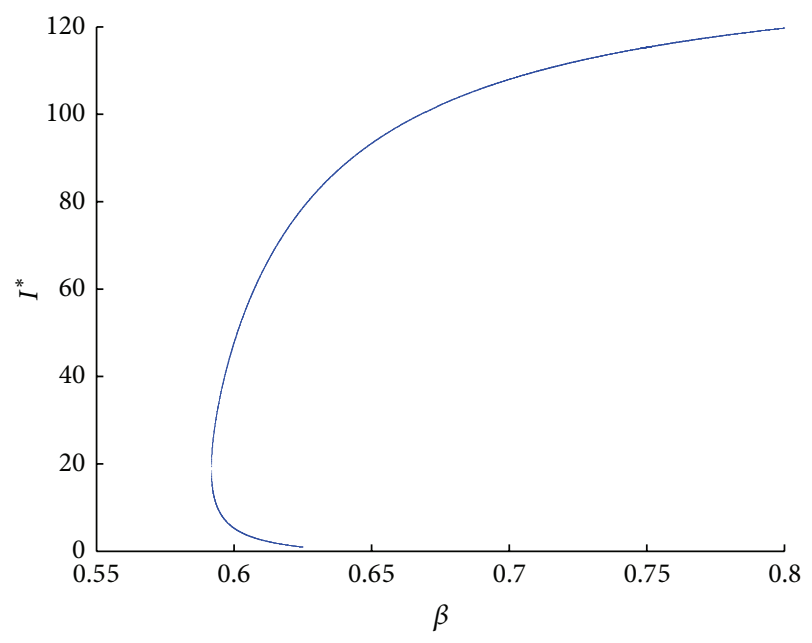

(b)

Figure 1: (a) Plot of the function $F(I)$ with different values of the transmission coefficient $\beta$. (b) Backward bifurcation when the transmission coefficient $\beta$ varies. Other parameters: $\Pi=100, \mu=1 / 45, w=1 / 20, \epsilon=0.4, \xi=0.6, \sigma_{1}=0.7, \sigma_{2}=0.4, \psi=0.5, q=1, h=1, \eta_{1}=0.4$, and $\eta_{2}=0.7$.

equilibria for $R_{c}<R_{0}<1$, and a unique endemic equilibrium exists for $R_{0}=R_{c}<1$ or $R_{0}>1$. Numerical studies will confirm this in the end of this subsection.

We now analyze the endemic equilibrium of model (1). The equilibrium of model (1) can be obtained as follows:

$$
\begin{aligned}
S^{*} & =\frac{\Pi\left((1-\epsilon) \lambda^{*}+\mu+\omega\right)}{\left((1-\epsilon) \lambda^{*}+\mu+\omega\right)\left(\lambda^{*}+\mu+\xi\right)-\omega \xi}, \\
V^{*} & =\frac{\Pi \xi}{\left((1-\epsilon) \lambda^{*}+\mu+\omega\right)\left(\lambda^{*}+\mu+\xi\right)-\omega \xi}, \\
D^{*} & =\frac{1}{\sigma_{2}+\mu} \frac{q I^{*}}{1+h I^{*}}, \\
A^{*} & =\frac{\sigma_{1}}{\mu+\psi} I^{*}+\frac{\sigma_{2}}{\mu+\psi} \frac{1}{\sigma_{2}+\mu} \frac{q I^{*}}{1+h I^{*}}, \\
N^{*} & =\frac{\Pi-\psi A^{*}}{\mu},
\end{aligned}
$$

where

$$
\begin{aligned}
\lambda^{*}=\lambda\left(I^{*}\right) & =\beta \frac{I^{*}+\eta_{1} D^{*}+\eta_{2} A^{*}}{N^{*}} \\
=\beta \frac{I^{*}}{N^{*}}\left\{1+\frac{\eta_{1}}{\sigma_{2}+\mu} \frac{q}{1+h I^{*}}\right. & \\
& \left.+\eta_{2}\left(\frac{\sigma_{1}}{\mu+\psi}+\frac{\sigma_{2}}{\mu+\psi} \frac{1}{\sigma_{2}+\mu} \frac{q}{1+h I^{*}}\right)\right\} .
\end{aligned}
$$

Substituting (20) into the third equation of system (1), it is easy to derive the following equation:

$$
f(I)=g(I),
$$

where

$$
\begin{gathered}
f(I)=(S+(1-\epsilon) V) \frac{\beta}{N} \\
\times\left\{1+\frac{\eta_{1}}{\sigma_{2}+\mu} \frac{q}{1+h I}\right. \\
\left.+\eta_{2}\left(\frac{\sigma_{1}}{\mu+\psi}+\frac{\sigma_{2}}{\mu+\psi} \frac{1}{\sigma_{2}+\mu} \frac{q}{1+h I}\right)\right\}, \\
g(I)=\sigma_{1}+\mu+\frac{q}{1+h I} .
\end{gathered}
$$

Clearly, $I^{*}=0$ is a fixed point, which corresponds to the disease-free equilibrium $E_{0}$. For $I=0$, we can obtain

$$
\begin{aligned}
g(0) & =\sigma_{1}+q+\mu, \\
f(0) & =\beta m\left\{1+\frac{\eta_{1} q}{\sigma_{2}+\mu}+\frac{\sigma_{2}}{\mu+\psi}\left(\sigma_{1}+\frac{\sigma_{2} q}{\sigma_{2}+\mu}\right)\right\}, \\
R_{0} & =\frac{f(0)}{g(0)} .
\end{aligned}
$$

Define

$$
F(I)=\frac{f(I)}{g(I)} .
$$

From model (1), it can be shown that if $I^{*}$ is a positive solution of $F(I)=1$, then $S^{*}, V^{*}, D^{*}$, and $A^{*}$ are positive. Thus, the equilibrium is biologically relevant. Unfortunately, it is hard to solve the equation $F(I)=1$ analytically; in the following we numerically show that this equation can have two positive roots, which confirms the existence of backward bifurcation. In Figure 1(a), $F(I)$ is plotted versus $I$ for different values of $\beta$ and all other parameters are fixed. Figure 1(a) shows that an increase in $\beta$ would lead to curve $F(I)$ becoming tangent 
to line 1 and defining a critical value $\left.F\left(I^{*}\right)\right|_{\beta=\beta_{c}}=1$ and $\left.F^{\prime}\left(I^{*}\right)\right|_{\beta=\beta_{c}}=0$ hold true. Figure $1(\mathrm{~b})$ shows the occurrence of the backward bifurcation as parameter $\beta$ varies. We write $R_{0}(\beta)$ as the threshold value to indicate $\beta$ as the bifurcation parameter while all other parameters are fixed. Define [16]

$$
R_{c}=R_{0}\left(\beta_{c}\right) \text {, }
$$

below which the disease-free equilibrium is unique equilibrium.

3.3. Stability Analysis of Equilibria. First, we have the following result on the local stability of $E_{0}$.

Theorem 2. The disease-free equilibrium $E_{0}$ of system (1) is locally asymptotically stable if $R_{0}<1$ and unstable otherwise.

Proof. By checking the Jacobian matrix of system (1) evaluated at $E_{0}$, we know that the characteristic equation for $J\left(E_{0}\right)$ has two eigenvalues as

$$
\lambda_{1}=-\mu, \quad \lambda_{2}=-(\mu+\omega+\xi),
$$

and the others satisfy the following equation:

$$
\begin{aligned}
h(\lambda)= & \frac{\beta m}{\lambda+\sigma_{1}+q+\mu}+\frac{\eta_{1} \beta m q}{\left(\lambda+\sigma_{1}+q+\mu\right)\left(\lambda+\sigma_{2}+\mu\right)} \\
& +\frac{\eta_{2} \beta m q \sigma_{2}}{\left(\lambda+\sigma_{1}+q+\mu\right)\left(\lambda+\sigma_{2}+\mu\right)(\lambda+\mu+\psi)} \\
& +\frac{\eta_{2} \beta m \sigma_{1}}{\left(\lambda+\sigma_{1}+q+\mu\right)(\lambda+\mu+\psi)}=1 .
\end{aligned}
$$

If the real parts of the roots of the equation $h(\lambda)=1$ are nonnegative, that is, $\mathfrak{R}(\lambda) \geq 0$, then [17]

$$
|h(\lambda)| \leq h(0)=R_{0}
$$

Hence, if $R_{0}<1, \forall \lambda$ such that $\Re(\lambda) \geq 0$, then $|h(\lambda)| \leq R_{0}<1$, showing that there are no solutions to $h(\lambda)=1$ with positive real part. Hence, $E_{0}$ is locally asymptotically stable if $R_{0}<1$. This proof is completed. of $E_{0}$.

Then, using Lyapunov function we can get global stability

Theorem 3. The disease-free equilibrium $E_{0}$ of system (1) is globally asymptotically stable if $R_{0}<\min \left\{R_{c},\left(\sigma_{1}+\mu\right) m /\left(\sigma_{1}+\right.\right.$ $q+\mu)\}$.

Proof. We note that no endemic equilibrium exists for $R_{0}<$ $R_{c}$. Then, $E_{0}$ is a unique equilibrium of system (1). We now consider a Lyapunov function:

$$
V=I+\frac{\beta}{\sigma_{2}+\mu}\left(\eta_{1}+\frac{\sigma_{2} \eta_{2}}{\mu+\psi}\right) D+\frac{\eta_{2} \beta}{\mu+\psi} A .
$$

The time derivative of $V$ is given by

$$
\begin{aligned}
& \dot{V}=\dot{I}+\frac{\beta}{\sigma_{2}+\mu}\left(\eta_{1}+\frac{\sigma_{2} \eta_{2}}{\mu+\psi}\right) \dot{D}+\frac{\eta_{2} \beta}{\mu+\psi} \dot{A} \\
& =\left\{\beta\left(I+\eta_{1} D+\eta_{2} A\right) \frac{S+(1-\epsilon) V}{N}-\sigma_{1} I-\frac{q I}{1+h I}-\mu I\right\} \\
& +\frac{\beta}{\sigma_{2}+\mu}\left(\eta_{1}+\frac{\sigma_{2} \eta_{2}}{\mu+\psi}\right)\left(\frac{q I}{1+h I}-\sigma_{2} D-\mu D\right) \\
& +\frac{\eta_{2} \beta}{\mu+\psi}\left(\sigma_{1} I+\sigma_{2} D-(\mu+\psi) A\right) \\
& \leq\left\{\beta\left(I+\eta_{1} D+\eta_{2} A\right)-\sigma_{1} I-\frac{q I}{1+h I}-\mu I\right\} \\
& +\frac{\beta}{\sigma_{2}+\mu}\left(\eta_{1}+\frac{\sigma_{2} \eta_{2}}{\mu+\psi}\right)\left(q I-\sigma_{2} D-\mu D\right) \\
& +\frac{\eta_{2} \beta}{\mu+\psi}\left(\sigma_{1} I+\sigma_{2} D-(\mu+\psi) A\right) \\
& =I\left\{\beta-\sigma_{1}-\frac{q}{1+h I}-\mu+\frac{\beta}{\sigma_{2}+\mu}\left(\eta_{1}+\frac{\sigma_{2} \eta_{2}}{\mu+\psi}\right) q\right. \\
& \left.+\frac{\eta_{2} \beta}{\mu+\psi} \sigma_{1}\right\} \\
& \leq I\left\{R_{0} \frac{\sigma_{1}+q+\mu}{m}-\left(\sigma_{1}+\mu\right)\right\} \\
& =\frac{\left(\sigma_{1}+q+\mu\right) I}{m}\left\{R_{0}-\frac{\left(\sigma_{1}+\mu\right) m}{\sigma_{1}+q+\mu}\right\} \text {. }
\end{aligned}
$$

Note that $\dot{V} \leq 0$ if $R_{0}<\left(\sigma_{1}+\mu\right) m /\left(\sigma_{1}+q+\mu\right)$. Furthermore, $\dot{V}=0$ if and only if $I=0$. Therefore, the largest compact invariant set in $\Omega: \dot{V}=0$, when $R_{0}<\min \left\{R_{c},\left(\sigma_{1}+\right.\right.$ $\left.\mu) m /\left(\sigma_{1}+q+\mu\right)\right\}$, is the singleton $E_{0}$. Thus, $E_{0}$ is globally asymptotically stable if $R_{0}<\min \left\{R_{c},\left(\sigma_{1}+\mu\right) m /\left(\sigma_{1}+q+\mu\right)\right\}$. This completes the proof.

\section{Persistence of the Model}

In this section, we will prove that system (1) is uniformly persistent. First, we present the following definition that is similar to that in $[18,19]$.

Definition 4. Model (1) is said to be uniformly persistent if there exists a positive constant $\varepsilon>0$ (independent of initial data) such that every solution with positive initial conditions satisfies $\liminf _{t \rightarrow \infty} S(t) \geq \varepsilon, \liminf _{t \rightarrow \infty} V(t) \geq$ $\varepsilon, \liminf _{t \rightarrow \infty} I(t) \geq \varepsilon, \liminf _{t \rightarrow \infty} D(t) \geq \varepsilon$, and $\liminf \operatorname{in}_{t \rightarrow \infty} A(t) \geq \varepsilon$.

Theorem 5. If $R_{0}>1$, system (1) is uniformly persistent; that is, there exists a positive constant $\varepsilon$, such that, for all initial values

$$
(S(0), V(0), I(0), D(0), A(0)) \in R_{+}^{2} \times \operatorname{Int}\left(R_{+}^{3}\right),
$$


the solutions of system (1) satisfy. $\liminf _{t \rightarrow \infty} S(t) \geq \varepsilon$, $\liminf _{t \rightarrow \infty} V(t) \geq \varepsilon, \liminf _{t \rightarrow \infty} I(t) \geq \varepsilon, \liminf _{t \rightarrow \infty} D(t) \geq$ $\varepsilon$, and $\liminf _{t \rightarrow \infty} A(t) \geq \varepsilon$.

Proof. Define

$$
\begin{aligned}
X & =\{(S, V, I, D, A) \mid S \geq 0, V \geq 0, I \geq 0, D \geq 0, A \geq 0\}, \\
X_{0} & =\{(S, V, I, D, A) \mid S \geq 0, V \geq 0, I>0, D>0, A>0\}, \\
\partial X_{0} & =X \backslash X_{0} .
\end{aligned}
$$

It then suffices to show that system (1) is uniformly persistent with respect to $\left(X_{0}, \partial X_{0}\right)$. First, by the form of (1), it is easy to see that both $X$ and $X_{0}$ are positively invariant. Clearly, $\partial X_{0}$ is relatively closed in $X$. Furthermore, system (1) is point dissipative. Set

$$
\begin{aligned}
M_{\partial}=\{( & (0), V(0), I(0), D(0), A(0)): \\
& (S(t), V(t), I(t), D(t), A(t)) \text { satisfies }(1), \\
& \left.(S(t), V(t), I(t), D(t), A(t)) \in \partial X_{0}, \forall t \geq 0\right\} .
\end{aligned}
$$

We now prove that

$$
M_{\partial}=\{(S, V, 0,0,0): S \geq 0, V \geq 0\} .
$$

Assume that $(S(0), V(0), I(0), D(0), A(0)) \in M_{\partial}$. It suffices to show that

$$
I(t)=0, \quad D(t)=0, \quad A(t)=0, \quad \forall t \geq 0 .
$$

If this is not true, then there exists a $t_{0} \geq 0$ such that

$$
I\left(t_{0}\right)>0, \quad D\left(t_{0}\right)=0, \quad A\left(t_{0}\right)=0,
$$

or

$$
I\left(t_{0}\right)>0, \quad D\left(t_{0}\right)>0, \quad A\left(t_{0}\right)=0,
$$

or

$$
I\left(t_{0}\right)>0, \quad D\left(t_{0}\right)=0, \quad A\left(t_{0}\right)>0,
$$

or

$$
I\left(t_{0}\right)=0, \quad D\left(t_{0}\right)>0, \quad A\left(t_{0}\right)=0,
$$

or

$$
I\left(t_{0}\right)=0, \quad D\left(t_{0}\right)=0, \quad A\left(t_{0}\right)>0,
$$

or

$$
I\left(t_{0}\right)=0, \quad D\left(t_{0}\right)>0, \quad A\left(t_{0}\right)>0 .
$$

For $I\left(t_{0}\right)>0, D\left(t_{0}\right)=0$, and $A\left(t_{0}\right)=0$, we get

$$
D^{\prime}\left(t_{0}\right)=\frac{q I\left(t_{0}\right)}{1+h I\left(t_{0}\right)}>0, \quad A^{\prime}\left(t_{0}\right)=\sigma_{1} I\left(t_{0}\right)>0 .
$$

It follows that there is an $\varepsilon_{0}>0$ such that $D(t)>0, A(t)>0$, for $t_{0}<t<t_{0}+\varepsilon_{0}$. This proves that

$$
(S(t), V(t), I(t), D(t), A(t)) \notin \partial X_{0} \quad \text { for } t_{0}<t<t_{0}+\varepsilon_{0},
$$

which contradicts the assumption that $(S(0), V(0), I(0)$, $D(0), A(0)) \in M_{\partial}$. Similarly, we can obtain contradictions for other cases. This proves that

$$
M_{\partial}=\{(S, V, 0,0,0): S \geq 0, V \geq 0\} .
$$

Note that $E_{0}$ is globally asymptotically stable in Int $M_{\partial}$, and $E_{0}$ is an isolated invariant set in $X$. That is to say, $W^{s}\left(E_{0}\right) \cap$ $X_{0}=\emptyset$. Every orbit in $M_{\partial}$ converges to $E_{0}$, and $E_{0}$ is acyclic in $M_{\partial}$. We claim that $W^{s}\left(E_{0}\right) \cap X_{0}=\emptyset$ for $R_{0}>1$. If this is false, then we have $W^{s}\left(E_{0}\right) \cap X_{0} \neq \emptyset$. The system has a positive solution

$$
(S(t), V(t), I(t), D(t), A(t)),
$$

where $(S(0), V(0), I(0), D(0), A(0)) \in X_{0}$. Then

$$
\begin{array}{r}
(S(t), V(t), I(t), D(t), A(t)) \longrightarrow E_{0} \\
\text { as } t \longrightarrow \infty \text { for } R_{0}>1 .
\end{array}
$$

For $R_{0}>1$, we can choose an $\eta>0$ small enough such that $R_{0}(1-\eta)>1$. Then, when $t$ is sufficiently large, we have

$$
\begin{gathered}
m-\eta m \leq \frac{S(t)+(1-\epsilon) V(t)}{N(t)} \leq m+\eta m, \\
I^{\prime} \geq \beta m(1-\eta)\left(I+\eta_{1} D+\eta_{2} A\right)-\sigma_{1} I-\frac{q I}{1+h I}-\mu I, \\
D^{\prime} \geq \frac{q I}{1+h I}-\sigma_{2} D-\mu D, \\
A^{\prime} \geq \sigma_{1} I+\sigma_{2} D-(\mu+\psi) A .
\end{gathered}
$$

Define

$M$

$$
=\left(\begin{array}{ccc}
\beta m(1-\eta)-\sigma_{1}-q-\mu & \eta_{1} \beta m(1-\eta) & \eta_{2} \beta m(1-\eta) \\
q & -\sigma_{2}-\mu & 0 \\
\sigma_{1} & \sigma_{2} & -\mu-\psi
\end{array}\right) .
$$

Recall that the stability modulus of an $n \times n$ matrix $M$, denoted by $s(M)$, is defined as

$$
s(M)=\max \{\operatorname{Re} \lambda: \lambda \text { is an eigenvalue of } M\} .
$$

Note that $M$ is irreducible and has nonnegative offdiagonal elements. It then follows that $s(M)$ is a simple eigenvalue of $M$ with a (componentwise) positive eigenvector. Thus,

$$
|\lambda I-M|=\lambda^{3}+a_{1} \lambda^{2}+a_{2} \lambda+a_{3},
$$


Table 1: Parameter description and values.

\begin{tabular}{|c|c|c|c|}
\hline Parameters & Description & Estimated values & Source \\
\hline$\Pi$ & Recruitment rate & 4348 & [21] \\
\hline$\beta$ & Transmission coefficient & 0.304 & {$[26]$} \\
\hline$w$ & Per capita waning rate of vaccine & $1 / 20$ & {$[5]$} \\
\hline$\xi$ & Per capita vaccination rate & 0.4 & Variable \\
\hline$\varepsilon$ & Vaccine efficacy & 0.4 & Variable \\
\hline$\mu$ & Natural death rate & 0.0246 & {$[27]$} \\
\hline$\psi$ & Disease-induced death rate & 0.7114 & {$[21]$} \\
\hline$\sigma_{1}$ & Progression rate to AIDS stage for the infection stage & 0.0413 & [9] \\
\hline$\sigma_{2}$ & Progression rate to AIDS stage for the diagnosed stage & 0.116 & [9] \\
\hline$q$ & Diagnosis rate & 0.304 & [9] \\
\hline$\eta_{1}$ & Modification factor in transmission coefficient of diagnosed HIV-positive individuals & 0.491 & {$[9]$} \\
\hline$\eta_{2}$ & Modification factor in transmission coefficient of AIDS patients & 0.1 & Variable \\
\hline
\end{tabular}

where

$$
\begin{gathered}
a_{1}=\left(\sigma_{1}+q+\mu\right)+\left(\sigma_{2}+\mu\right)+(\mu+\psi)-\beta m, \\
a_{2}=\left(\sigma_{1}+q+\mu-\beta m\right)\left(\sigma_{2}+\mu\right)+\left(\sigma_{2}+\mu\right)(\mu+\psi) \\
\quad+\left(\sigma_{1}+q+\mu-\beta m\right)(\mu+\psi)-\eta_{1} \beta m q-\eta_{2} \beta m \sigma_{1}, \\
a_{3}=\left(\sigma_{1}+q+\mu\right)\left(\sigma_{2}+\mu\right)(\mu+\psi)\left(1-R_{0}(1-\eta)\right) .
\end{gathered}
$$

For $R_{0}(1-\eta)>1$, we obtain $a_{3}<0$. Thus, $s(M)$ is a simple positive eigenvalue of $M$ with a (componentwise) positive eigenvector. By comparison theorem, we get

$$
\begin{aligned}
I(t) \longrightarrow \infty, \quad D(t) \longrightarrow \infty, \quad & A(t) \longrightarrow \infty, \\
& \text { as } t \longrightarrow \infty,
\end{aligned}
$$

which contradicts the assumption that

$$
(S(t), V(t), I(t), D(t), A(t)) \longrightarrow E_{0} \quad \text { as } t \longrightarrow \infty \text {. }
$$

This proves that $W^{s}\left(E_{0}\right) \cap X_{0}=\emptyset$ for $R_{0}>1$. By [20], system (1) is uniformly persistent. Thus, the proof of the theorem is completed.

\section{Numerical Simulations}

5.1. Numerical Results. We initially investigate variation in $R_{0}$ with different vaccine efficacy, vaccination rate, and diagnosis rate to compare the impact of these intervention measures on HIV transmission. The parameter values in Table 1 are chosen based on HIV/AIDS transmission among IDUs in Yunnan province, China. For simplicity, we choose $h=1$. Differentiating partially $R_{0}$ with respect to $\xi$ and $\epsilon$, respectively, we obtain

$$
\begin{aligned}
\frac{\partial R_{0}}{\partial \xi}= & -\frac{\beta}{\sigma_{1}+q+\mu} \frac{\epsilon(\mu+\omega)}{(\mu+\omega+\xi)^{2}} \\
& \times\left(1+\frac{\eta_{1} q}{\sigma_{2}+\mu}+\frac{\eta_{2}}{\mu+\psi}\left(\frac{\sigma_{2} q}{\sigma_{2}+\mu}+\sigma_{1}\right)\right)<0, \\
\frac{\partial R_{0}}{\partial \epsilon}= & -\frac{\beta}{\sigma_{1}+q+\mu} \frac{\xi}{\mu+\omega+\xi} \\
& \times\left(1+\frac{\eta_{1} q}{\sigma_{2}+\mu}+\frac{\eta_{2}}{\mu+\psi}\left(\frac{\sigma_{2} q}{\sigma_{2}+\mu}+\sigma_{1}\right)\right)<0,
\end{aligned}
$$

which implies that an increase of vaccination rate and vaccine efficacy leads to the basic reproduction number decline, as shown in Figure 2(a), in which the contour plots of $R_{0}$ versus vaccine efficacy $\epsilon$ and vaccination rate $\xi$ were plotted. It also shows that the basic reproduction number is more sensitive to vaccine efficacy than vaccination rate. Figure 2(b) shows the contour plot of $R_{0}$ with diagnosis rate and vaccination rate, which implies a decrease in $R_{0}$ with increasing diagnosis rate $q$ and vaccination rate $\xi$. Furthermore, when $50 \%$ of HIV individuals are diagnosed, vaccination level of at least $60 \%$ would be needed to achieve $R_{0}<1$. This suggests that the strategies of diagnosis and vaccination should be stringent enough to reduce $R_{0}$.

Next, we consider the effect of different transmission rate, vaccination rate, vaccine efficacy, and recruitment rate on transmission of HIV/AIDS. We take the year 2004 as starting time; since then the policy of diagnosis is consistent. In [21], we get that the number of diagnosed HIV-positive individuals and AIDS patients in Yunnan province was 27168 and 1223 in year 2004, respectively. Besides, $22.6 \%$ of these HIV individuals were transmitted by share injectors [22]. Hence, $D(0)=27168 \times 22.6 \%=6140, A(0)=1223 \times 22.6 \%=$ 276. Note that the diagnosis rate is estimated to be 0.304 [9]; then we have $I(0)=D(0) / 0.304=20197$. We have no reliable data on the number of susceptible individuals, that is, number of IDUs in Yunnan province. However, we know 


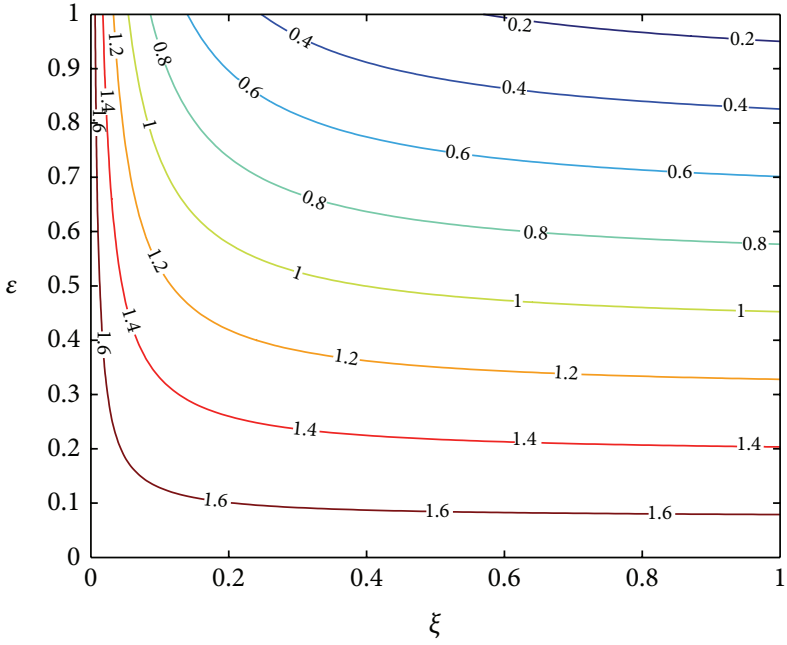

(a)

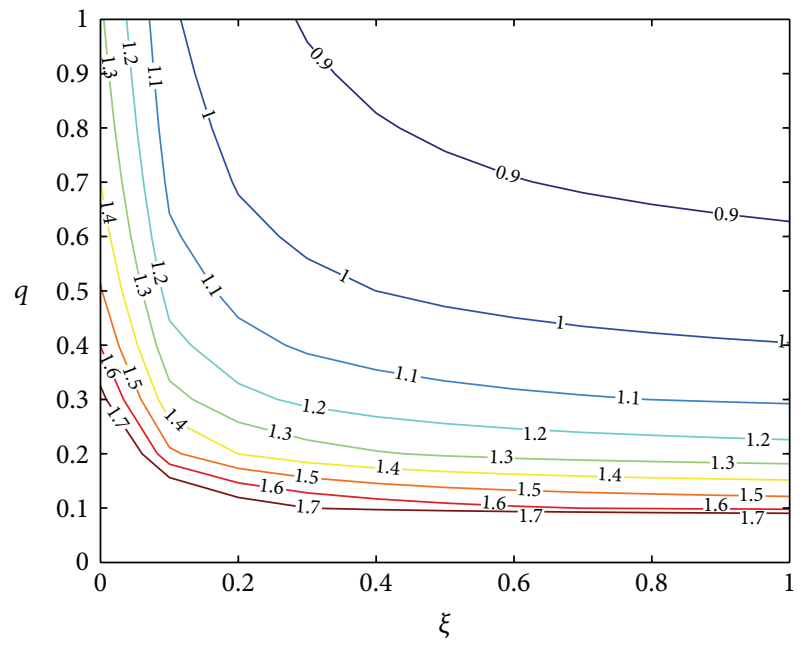

(b)

Figure 2: Contour plots of $R_{0}$ versus (a) vaccine efficacy $\epsilon$ and vaccination rate $\xi$ and (b) diagnosis rate $q$ and vaccination rate $\xi$.

that 3.2 million blood samples were tested in Yunnan in [23]. We then assume that in these blood samples the fraction of share injectors is the same as fraction of transmission via share injectors (i.e., 22.6\%). Then the number of susceptible individuals who share injectors is $S(0)+V(0)=3.2 \times 10^{6} \times$ $22.6 \%=723200$. If the vaccination rate is assumed to be 0.4 , then $V(0)=S(0) \times 0.4$. We obtain the initial values

$$
\begin{gathered}
S(0)=516571, \quad V(0)=206628, \quad I(0)=20197, \\
D(0)=6140, \quad A(0)=276 .
\end{gathered}
$$

Figure 3 shows the variation in the number of HIV infected individuals with different transmission rates, vaccination rates, vaccine efficacy, and recruitment rates. It follows from Figure 3(a) that decreasing transmission rate could lead to the number of HIV-positive individuals decline. The effect of increasing vaccination rate on HIV transmission is shown in Figure 3(b) and it is seen that the number of HIVpositive individuals becomes much smaller if vaccination rate increases more. Figure 3(c) illustrates that, with increasing vaccine efficacy, the number of HIV-positive individuals decreases. Figure 3(d) shows that if the inflow of susceptible individuals into the community is restricted due to education, the disease spread will slow down.

5.2. Sensitivity Analysis. In this section, we use sensitivity analysis method [24] to investigate the impact of various intervention measures on HIV transmission in Yunnan province, China. We hope that these results obtained here could improve the knowledge of the effects of different interventions.

Figures 4(a) and 4(b) show the comparison of sensitivity coefficients of new cases and prevalence against parameters $\beta$, $\epsilon, \xi, q$, and $\Pi$, separately. Note that the sensitivity coefficient of new cases and prevalence can be interpreted as the percentage change in the number of new cases and prevalence for $1 \%$ decline in the parameters $\beta$ and $\Pi$ or $1 \%$ increase in $\epsilon, \xi$, and $q$, respectively [25]. In particular, let function $f$ be new cases or prevalence; the sensitivity coefficients (SC) of new cases and prevalence are given by

SC $=\frac{f(\text { perturbed variables })-f(\text { original variable })}{f(\text { original variable })} \times 100 \%$.

It follows from Figure 4 that a decrease in transmission coefficient $\beta$ causes new cases and prevalence decline substantially. Besides, an increase in vaccine efficacy $\epsilon$ and vaccination rate $\xi$ can lead to a decrease in new cases and prevalence, whereas the change of both diagnosis rate $q$ and recruitment rate $\Pi$ slightly affects the new cases or prevalence. Thus, new cases and prevalence are sensitive to transmission coefficient, vaccine efficacy, and vaccination rate. Then, reducing the transmission coefficient and increasing the vaccine efficacy and vaccination rate can greatly reduce new cases and prevalence.

\section{Conclusion}

In this paper, we established an epidemic model to investigate effects of saturated diagnosis and vaccination on HIV transmission. It proved that backward bifurcation occurs by employing center manifold theory, which causes the diseasefree equilibrium to be locally asymptotically stable instead of globally asymptotically stable for $R_{0}<1$. Thus, making the basic reproduction number less than unity is not enough to eliminate the HIV infection. We note that $R_{0}<1$ is equivalent to

$$
\begin{aligned}
\xi>((\mu+\omega)[ & \beta\left(1+\frac{\eta_{1} q}{\sigma_{2}+\mu}+\frac{\eta_{2}}{\mu+\psi}\left(\frac{\sigma_{2} q}{\sigma_{2}+\mu}+\sigma_{1}\right)\right) \\
& \left.\left.-\left(\sigma_{1}+q+\mu\right)\right]\right)
\end{aligned}
$$




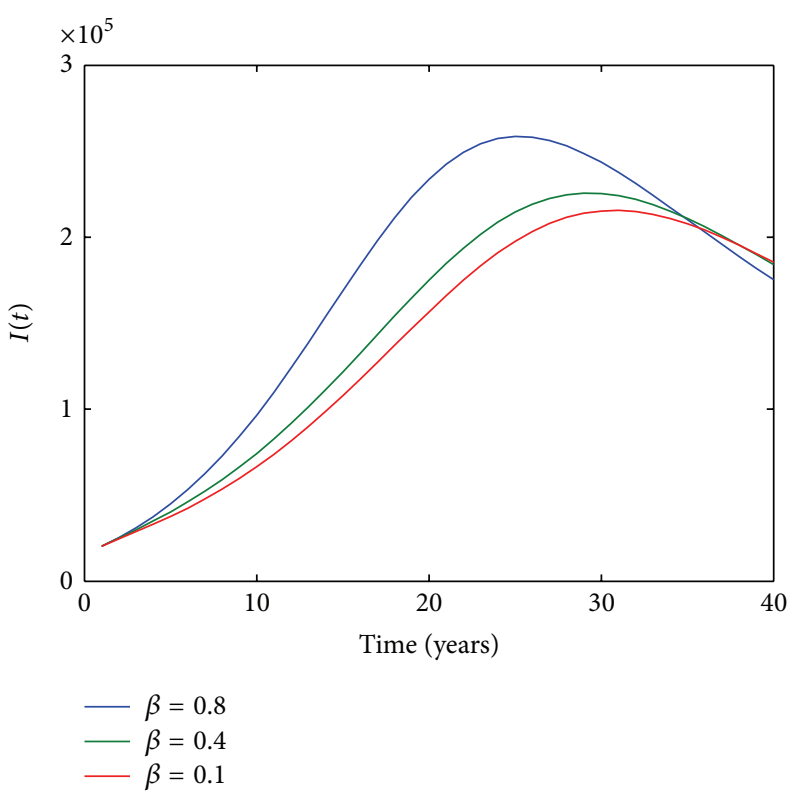

(a)

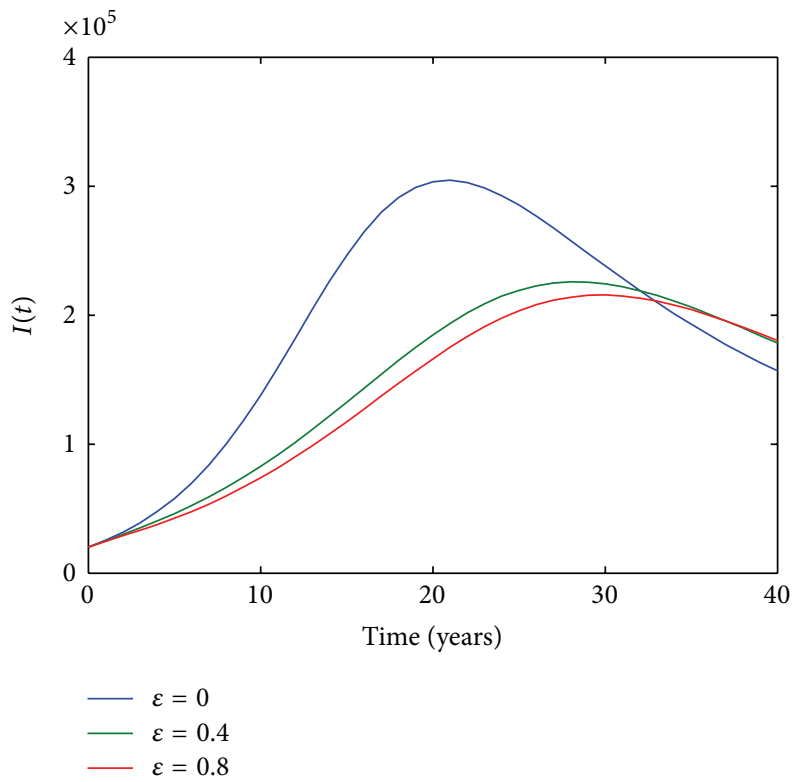

(c)

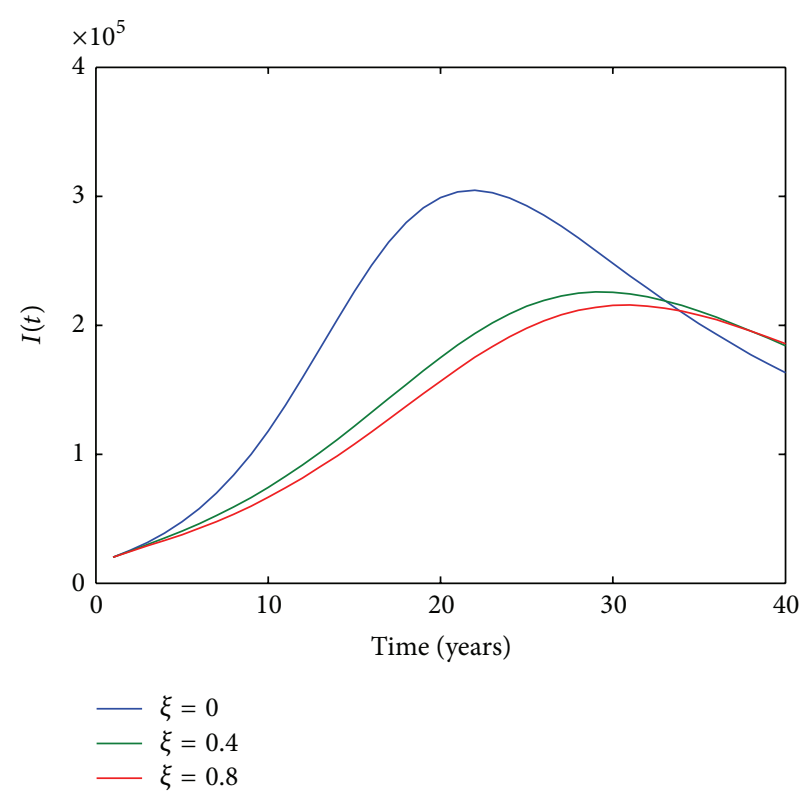

(b)

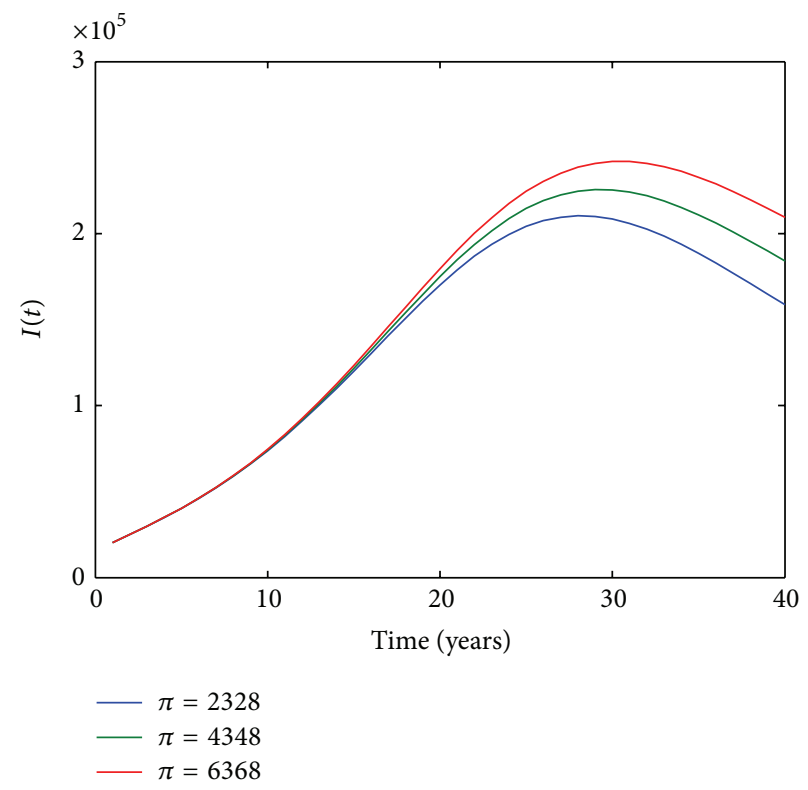

(d)

FIGURE 3: Variation in the number of HIV-positive individuals with different (a) transmission coefficient, (b) vaccination rate, (c) vaccine efficacy, and (d) recruitment rate.

$$
\begin{aligned}
& \quad \times\left(\left(\sigma_{1}+q+\mu\right)\right. \\
& \left.\quad-\beta(1-\epsilon)\left(1+\frac{\eta_{1} q}{\sigma_{2}+\mu}+\frac{\eta_{2}}{\mu+\psi}\left(\frac{\sigma_{2} q}{\sigma_{2}+\mu}+\sigma_{1}\right)\right)\right)^{-1} \\
& \quad:=\xi_{c},
\end{aligned}
$$

which means that only the vaccination rate is greater than $\xi_{c}$; HIV infection might be eliminated, depending on initial data. There exists the critical threshold $R_{c}$, which cannot be explicitly expressed due to nonlinearity, such that when $R_{0}<\min \left\{R_{c},\left(\sigma_{1}+\mu\right) m /\left(\sigma_{1}+q+\mu\right)\right\}<1$, the diseasefree equilibrium is globally asymptotically stable. However, if $R_{0}>1$, the disease uniformly persists.

It is interesting to note that if the diagnosis is described linearly backward bifurcation does not happen. This implies that nonlinear diagnosis due to limited medical resources leads to backward bifurcation, and consequently complete elimination of HIV infection becomes difficult. That is, HIV infection might be extinct only by improving integrated interventions, which ensures that $R_{0}$ is less than $R_{c}$ and $\left(\sigma_{1}+\right.$ $\mu) m /\left(\sigma_{1}+q+\mu\right)$. 


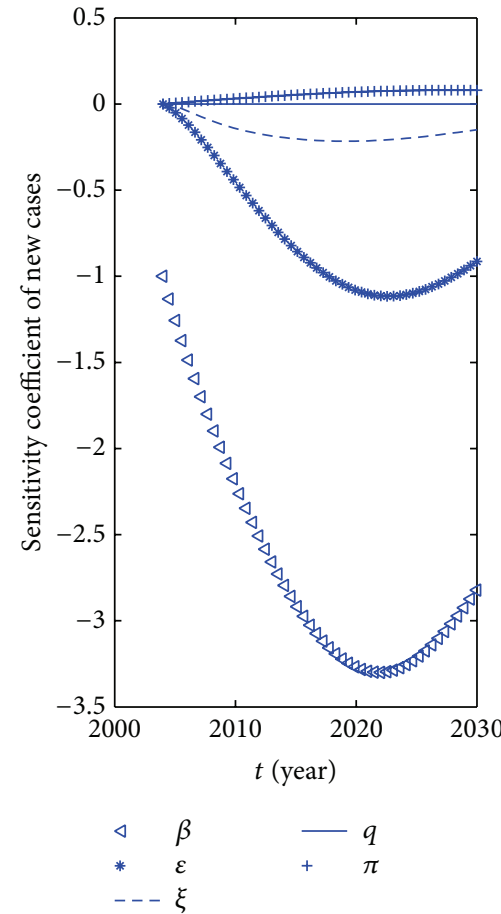

(a)

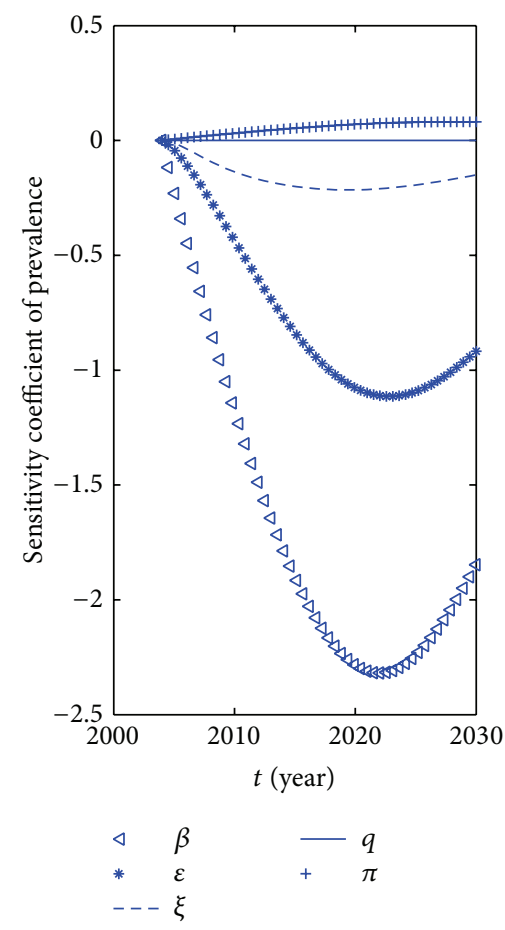

(b)

FIgURE 4: Sensitivity coefficients of new cases (a) and prevalence (b) on $\beta, \epsilon, \xi, q$, and $\Pi$ over time $t$. All other parameters are shown in Table 1 .

Since several candidate HIV vaccines are in development, it is useful to study the effectiveness. Moreover, the detection of HIV-positive individuals is limited due to medical resources. We then applied the proposed model with nonlinear diagnosis and vaccination to examine HIV infection among IDUs in Yunnan province, China. Sensitivity analysis shows that new cases and prevalence are sensitive to transmission rate, vaccine efficacy, and vaccination rate, whereas diagnosis rate and recruitment rate slightly affect both of them. Therefore, enlarging vaccination rate, improving vaccine efficacy, and lowering transmission rate by reducing sterile injecting equipment are beneficial to reduce transmission of HIV infection. In order to efficiently reduce HIV transmission, combined intervention strategies are suggested to be implemented simultaneously.

Effective antiretroviral therapy (ART) is an important strategy to slow down the progression to AIDS due to great reduction in viral loads and is not included in our model. Note that when HIV infected individuals are diagnosed and $\mathrm{CD} 4 \mathrm{~T}$ cell counts decrease to 350 copies $/ \mu \mathrm{L}$, they will accept treatment. We will include treatment strategy to construct HIV/AIDS models to investigate the transmission of HIV/AIDS in the future work and provide policy makers with effective suggestions.

\section{Conflict of Interests}

The authors declare that there is no conflict of interests regarding the publication of this paper.

\section{Acknowledgments}

The authors are supported by the National Megaproject of Science Research no. 2012ZX10001-001, the National Natural Science Foundation of China (NSFC, 11171268 (YX)), the Fundamental Research Funds for the Central Universities (GK 08143042 (YX)), and the International Development Research Center, Ottawa, Canada (104519-010).

\section{References}

[1] J. M. Hyman, J. Li, and E. A. Stanley, "The differential infectivity and staged progression models for the transmission of HIV," Mathematical Biosciences, vol. 155, no. 2, pp. 77-109, 1999.

[2] R. Naresh, A. Tripathi, and D. Sharma, "Modelling and analysis of the spread of AIDS epidemic with immigration of HIV infectives," Mathematical and Computer Modelling, vol. 49, no. 5-6, pp. 880-892, 2009.

[3] F. Nyabadza, Z. Mukandavire, and S. D. Hove-Musekwa, "Modelling the HIV/AIDS epidemic trends in South Africa: insights from a simple mathematical model," Nonlinear Analysis: Real World Applications, vol. 12, no. 4, pp. 2091-2104, 2011.

[4] J. M. Hyman, J. Li, and E. A. Stanley, "Modeling the impact of random screening and contact tracing in reducing the spread of HIV," Mathematical Biosciences, vol. 181, no. 1, pp. 17-54, 2003.

[5] A. B. Gumel, C. C. McCluskey, and P. van den Driessche, "Mathematical study of a staged-progression HIV model with imperfect vaccine," Bulletin of Mathematical Biology, vol. 68, no. 8, pp. 2105-2128, 2006.

[6] E. H. Elbasha and A. B. Gumel, "Theoretical assessment of public health impact of imperfect prophylactic HIV-1 vaccines 
with therapeutic benefits," Bulletin of Mathematical Biology, vol. 68 , no. 3, pp. 577-614, 2006.

[7] O. Sharomi, C. N. Podder, A. B. Gumel, E. H. Elbasha, and J. Watmough, "Role of incidence function in vaccine-induced backward bifurcation in some HIV models," Mathematical Biosciences, vol. 210, no. 2, pp. 436-463, 2007.

[8] F. Nyabadza and Z. Mukandavire, "Modelling HIV/AIDS in the presence of an HIV testing and screening campaign," Journal of Theoretical Biology, vol. 280, no. 1, pp. 167-179, 2011.

[9] Y. Xiao, S. Tang, Y. Zhou, R. J. Smith, J. Wu, and N. Wang, "Predicting the HIV/AIDS epidemic and measuring the effect of mobility in mainland China," Journal of Theoretical Biology, vol. 317, pp. 271-285, 2013.

[10] X. Zhang and X. Liu, "Backward bifurcation of an epidemic model with saturated treatment function," Journal of Mathematical Analysis and Applications, vol. 348, no. 1, pp. 433-443, 2008.

[11] P. van den Driessche and J. Watmough, "Reproduction numbers and sub-threshold endemic equilibria for compartmental models of disease transmission," Mathematical Biosciences, vol. 180, no. 1-2, pp. 29-48, 2002.

[12] C. Castillo-Chavez and B. Song, "Dynamical models of tuberculosis and their applications," Mathematical Biosciences and Engineering, vol. 1, no. 2, pp. 361-404, 2004.

[13] B. Buonomo and D. Lacitignola, "On the backward bifurcation of a vaccination model with nonlinear incidence," Nonlinear Analysis: Modelling and Control, vol. 16, no. 1, pp. 30-46, 2011.

[14] S. D. Hove-Musekwa, F. Nyabadza, and H. MambiliMamboundou, "Modelling hospitalization, home-based care, and individual withdrawal for people living with HIV/AIDS in high prevalence settings," Bulletin of Mathematical Biology, vol. 73, no. 12, pp. 2888-2915, 2011.

[15] L. Zhou and M. Fan, "Dynamics of an SIR epidemic model with limited medical resources revisited," Nonlinear Analysis: Real World Applications, vol. 13, no. 1, pp. 312-324, 2012.

[16] J. Arino, C. C. McCluskey, and P. van den Driessche, "Global results for an epidemic model with vaccination that exhibits backward bifurcation," SIAM Journal on Applied Mathematics, vol. 64, no. 1, pp. 260-276, 2003.

[17] Z. Mukandavire and W. Garira, "Effects of public health educational campaigns and the role of sex workers on the spread of HIV/AIDS among heterosexuals," Theoretical Population Biology, vol. 72, no. 3, pp. 346-365, 2007.

[18] Y. Kuang, Delay Differential Equations with Applications in Population Dynamics, vol. 191 of Mathematics in Science and Engineering, Academic Press, Boston, Mass, USA, 1993.

[19] Y. Xiao and L. Chen, "Modeling and analysis of a predator-prey model with disease in the prey," Mathematical Biosciences, vol. 171, no. 1, pp. 59-82, 2001.

[20] X.-Q. Zhao, Dynamical Systems in Population Biology, CMS Books in Mathematics/Ouvrages de Mathématiques de la SMC, 16, Springer, New York, NY, USA, 2003.

[21] T. Zhang, M. Jia, H. Luo, Y. Zhou, and N. Wang, "Study on a HIV/AIDS model with application to Yunnan province, China," Applied Mathematical Modelling, vol. 35, no. 9, pp. 4379-4392, 2011.

[22] L. Lu, M. Jia, J. Lu, H. Luo, and X. Zhang, "Analysis of HIV/AIDS prevalence in Yunnan province," Journal for China AIDS/STD, vol. 11, no. 3, pp. 172-175, 2005.

[23] L. Lu, M. Jia, Y. Ma et al., "The changing face of HIV in China," Nature, vol. 455, no. 7213, pp. 609-611, 2008.
[24] X. Xu, Y. Xiao, and N. Wang, "Modeling sexual transmission of HIV/AIDS in Jiangsu province, China," Mathematical Methods in the Applied Sciences, vol. 36, no. 2, pp. 234-248, 2013.

[25] L. Liu, X.-Q. Zhao, and Y. Zhou, "A tuberculosis model with seasonality," Bulletin of Mathematical Biology, vol. 72, no. 4, pp. 931-952, 2010.

[26] L. Han, J. Lou, Y. Ruan, and Y. Shao, "The analysis of the HIV/AIDS mathematical model for the injection drug use population," Journal of Biomathematics, vol. 23, no. 3, pp. 429434, 2008.

[27] N. Bacaër, X. Abdurahman, and J. Ye, "Modeling the HIV/AIDS epidemic among injecting drug users and sex workers in Kunming, China," Bulletin of Mathematical Biology, vol. 68, no. 3, pp. 525-550, 2006. 


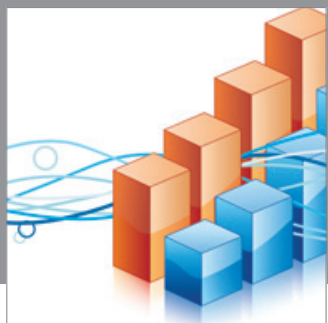

Advances in

Operations Research

mansans

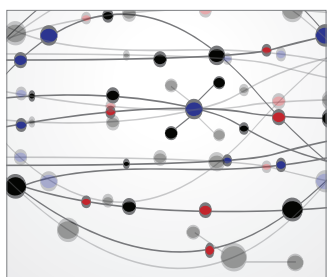

The Scientific World Journal
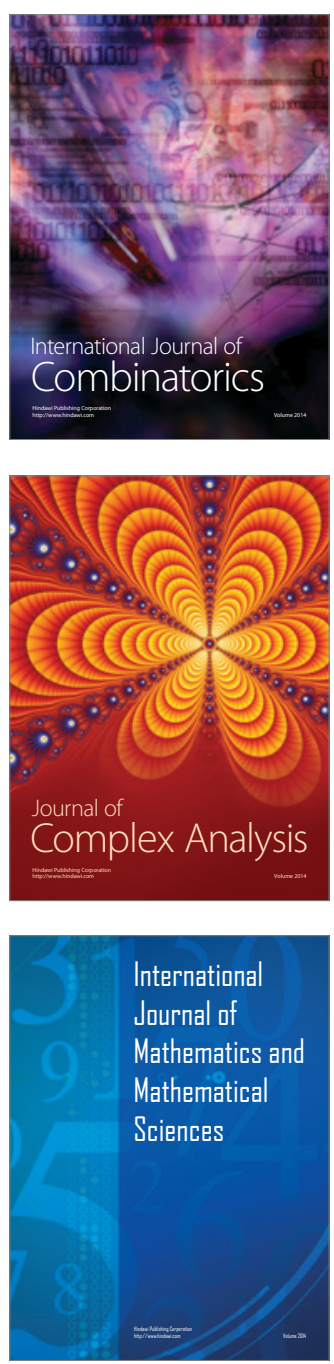
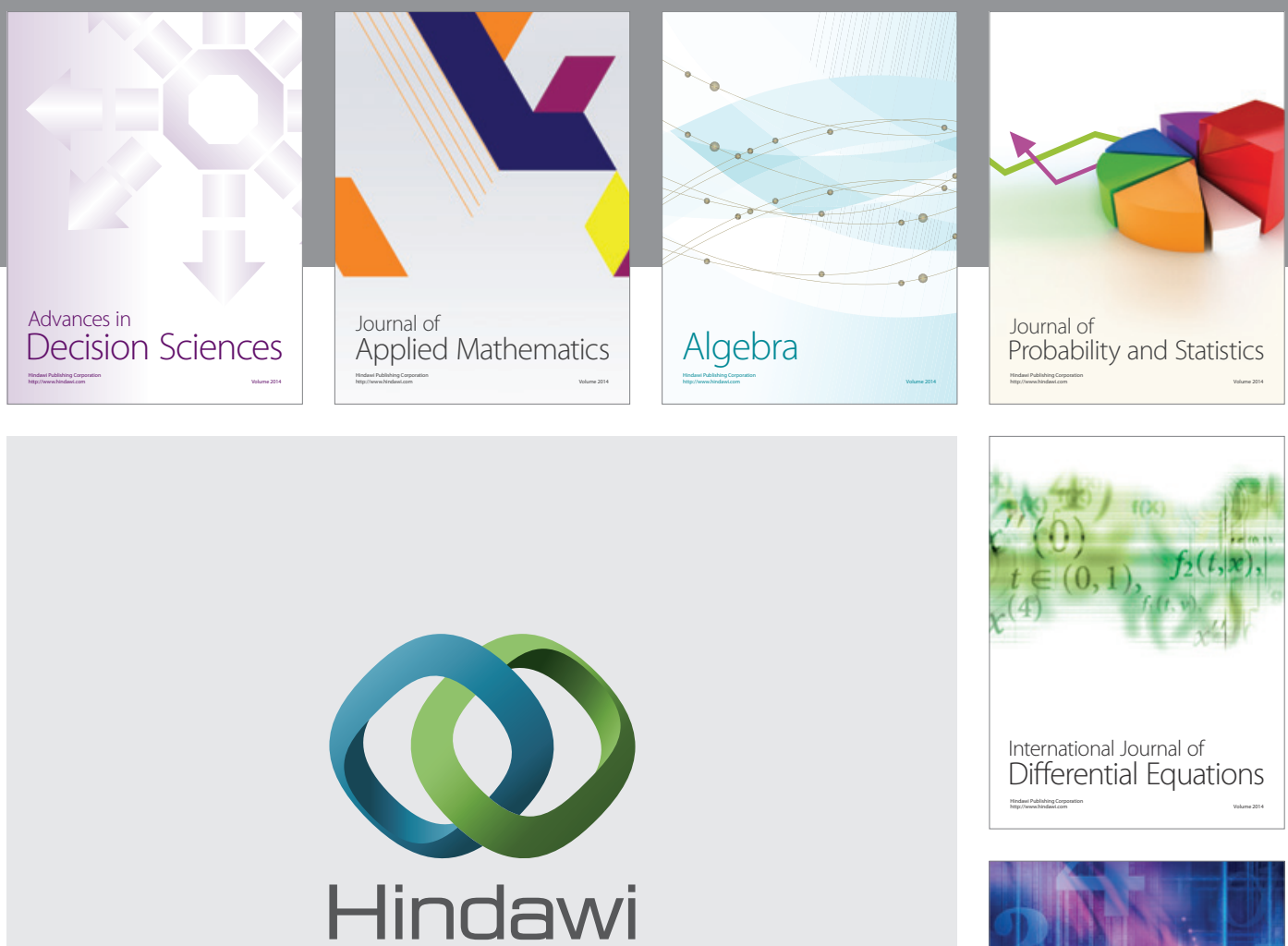

Submit your manuscripts at http://www.hindawi.com
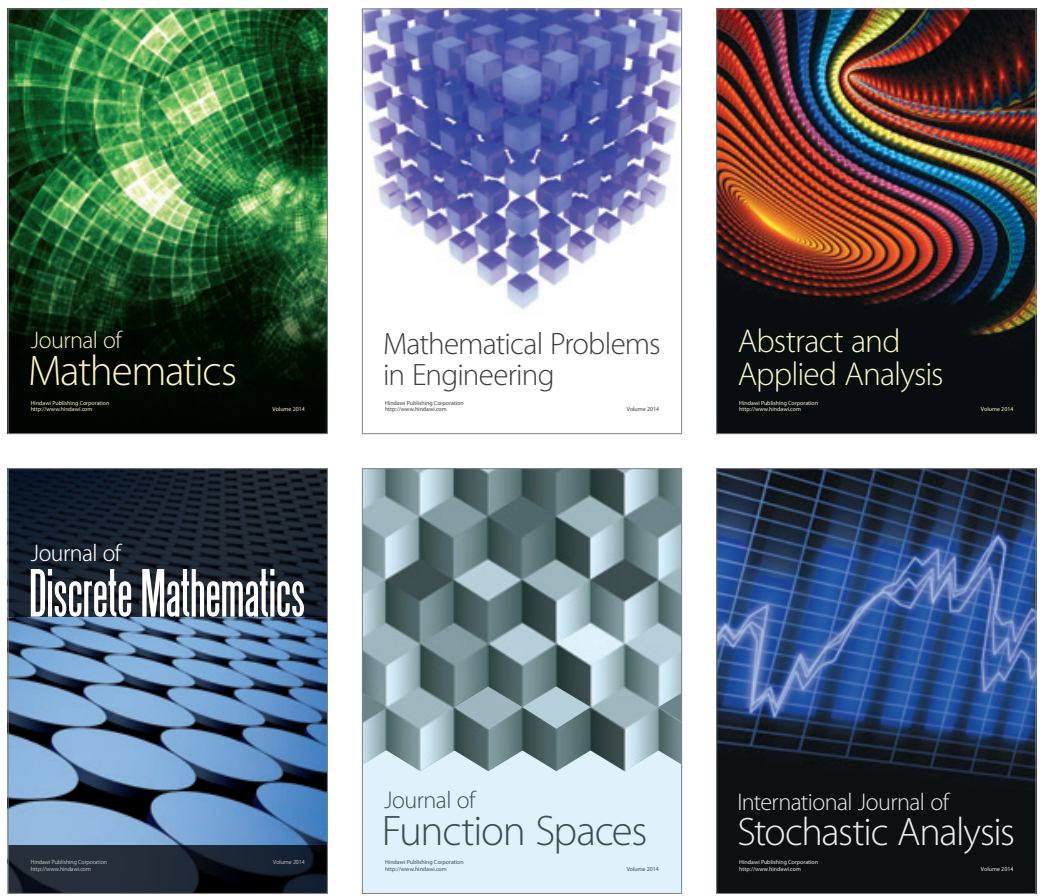

Journal of

Function Spaces

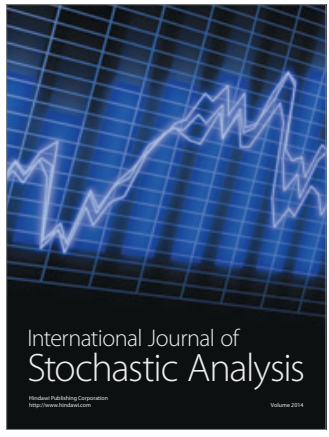

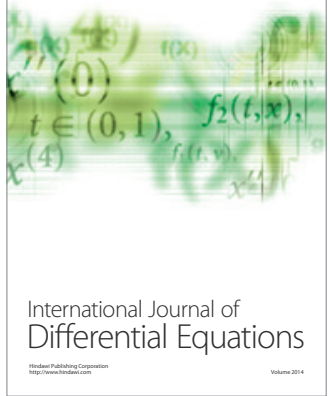
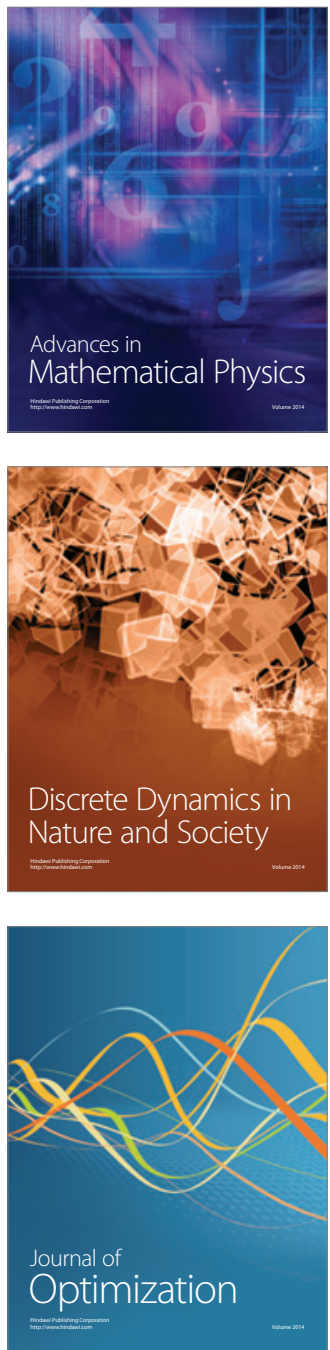